\title{
Welcher Beatmungszugang für welchen Patienten auf der Intensivstation?
}

\author{
Airway Devices in the Intensive Care Unit
}

Autoren

Institute
M. Dreher ${ }^{1}$, S. Kluge $^{2}$

Sektion Pneumologie der Klinik für Kardiologie, Pneumologie, Angiologie und internistische Intensivmedizin, Universitätsklinikum Aachen

' Klinik für Intensivmedizin, Universitätsklinikum Hamburg-Eppendorf
Bibliografie

DOI http://dx.doi.org/

10.1055/s-0034-1365318

Online-Publikation: 25.3.2014

Pneumologie 2014; 68: 371-377

(c) Georg Thieme Verlag KG

Stuttgart · New York

ISSN 0934-8387

\section{Korrespondenzadresse}

PD Dr. med. Stefan Kluge

Klinik für Intensivmedizin

Universitätsklinikum

Hamburg-Eppendorf

Martinistr. 52

20246 Hamburg

skluge@uke.de

Serienherausgeber

W. Windisch, Köln

\section{Zusammenfassung \\ $\nabla$}

Dieser Artikel gibt einen Überblick über die derzeit auf der Intensivstation eingesetzten Beatmungszugänge. Bei der nichtinvasiven Beatmung liegt der Beatmungszugang definitionsgemäß außerhalb des Körpers, wodurch in der Regel keine Analgosedierung vonnöten ist. Hierdurch ist eine gewisse Kooperation vonseiten des Patienten nötig, welches eine sorgfältige Auswahl des Beatmungszuganges voraussetzt. Die Maskenintoleranz stellt das häufigste Problem für das Versagen der nichtinvasiven Beatmung dar. Der am häufigsten verwendete Beatmungszugang bei der nichtinvasiven Beatmung auf der Intensivstation ist die Nasen-Mundmaske. Diese erlaubt dem Patienten eine, bei der akuten respiratorischen Insuffizienz oft notwendige, Mundatmung. Gute Alternativen sind die Vollgesichtsmaske und der Beatmungshelm. Nasenmasken oder Mundstücke werden auf der Intensivstation hingegen nur selten eingesetzt. Die Koniotomie und supraglottische Atemwegshilfen kommen als Notfallmaßnahme beim schwierigen Atemweg auf der Intensivstation zum Einsatz. Der Goldstandard zur Sicherung der Atemwege ist jedoch nach wie vor die endotracheale Intubation, welche mit einer Reihe von potentiellen Komplikationen assoziiert ist. Die Tracheotomie ist der Standardzugang, wenn eine Langzeitbeatmung beim Intensivpatienten absehbar wird.

\section{Einleitung}

$\nabla$

Die Beatmung bei respiratorischer Insuffizienz mit Sicherstellung einer adäquaten Oxygenierung und Ventilation ist eine der elementaren Aufgaben der Intensivmedizin. Dabei nimmt die Zahl der nichtinvasiv und invasiv beatmeten Patienten in den letzten Jahren kontinuierlich zu [1]. Der Beatmungszugang verbindet hierbei die anatomischen Luftwege des Patienten mit dem Be-

\section{Abstract \\ $\nabla$}

This review presents the potential indications for different airway devices in the intensive care unit (ICU). Since the interface during noninvasive ventilation is located outside the body, sedation is not regularly needed. Therefore, selection of a fitting mask is essential to avoid mask intolerance which is one of the biggest problems during noninvasive ventilation. Full-face masks are the interfaces mostly used for noninvasive ventilation to treat acute respiratory failure; here, mouth breathing is possible which is often necessary during acute respiratory failure. Good alternatives are total face masks and the helmet, whereas nasal masks and mouthpieces are not regularly used on the ICU. Extraglottic airway devices and coniotomy are used for the management of difficult airway situations on the ICU. Endotracheal intubation remains the gold standard procedure for airway management. However, this procedure is associated with a number of complications. Tracheostomy is the airway access of choice when longterm mechanical ventilation is necessary.

atmungsgerät und ist damit ein wesentlicher Bestandteil, der oft über Erfolg oder Misserfolg einer Beatmungstherapie entscheidet.

Während bei der invasiven Beatmung Endotrachealtuben und Trachealkanülen eingesetzt werden, stellen Nasen- und Nasen-Mundmasken die am häufigsten verwendeten Beatmungszugänge bei der nichtinvasiven Beatmung dar. In einigen wenigen Ausnahmen kommen transnasal eingelegte Endotrachealtuben bei der invasiven Beat- 
mung oder Mundstücke und Beatmungshelme bei der nichtinvasiven Beatmung zum Einsatz. Dieser Artikel soll einen Überblick über die derzeit auf der Intensivstation eingesetzten Beatmungszugänge geben.

\section{Beatmungszugänge bei nichtinvasiver Beatmung} $\nabla$

Unter nichtinvasiver Beatmung versteht man eine mechanische Ventilation der Lungen ohne Zuhilfenahme künstlicher, endotracheal liegender Beatmungszugänge. Somit liegt der Beatmungszugang, meistens die Beatmungsmaske, außerhalb des Körpers und umschließt in der Regel die Nase und/oder den Mund. Beatmungsmasken werden mittels Haltebändern am Kopf fixiert. Im Gegensatz zur invasiven Beatmung mit geblocktem Tubus oder geblockter Trachealkanüle sind bei der nichtinvasiven Beatmung die tiefen Atemwege nicht gesichert, weshalb der Einsatz einer nichtinvasiven Beatmung nur bei wachen Patienten mit vorhandenen Schutzreflexen (Schluck- und Würgereflex) erfolgen darf [2]. Diesbezüglich ist es umso wichtiger, einen Beatmungszugang zu finden, der einerseits eine adäquate Ventilation gewährleistet, andererseits aber auch von dem Patienten toleriert wird. Die Maskenintoleranz stellt ein großes Problem bei der nichtinvasiven Beatmung dar und ist einer der häufigsten Gründe für ein Therapieversagen. Weitere Probleme der Maskenbeatmung sind hohe Leckagen, welche eine effektive Therapie teilweise unmöglich machen oder aber mit Nebenwirkungen, wie z.B. einer Konjunktivitis, verbunden sind. Auf der anderen Seite führen maskenassoziierte Druckstellen nicht selten zu einem Therapieabbruch. Besonderes Augenmerk sollte auf die Kombination der verwendeten Maske und des Schlauchsystems gelegt werden, um eine adäquate $\mathrm{CO}_{2}$-Elimination zu gewährleisten. Den perfekten Beatmungszugang für die nichtinvasive Beatmung gibt es nicht, sodass die Wahl des Beatmungszuganges eine sorgfältige Beurteilung verschiedener Faktoren wie Patiententyp, Beatmungsmodus und Art der akuten respiratorischen Insuffizienz beinhaltet [3].

Am häufigsten werden sogenannte Nasenmasken oder NasenMundmasken eingesetzt. Da mittlerweile eine Vielzahl industriell gefertigter Masken in unterschiedlichsten Größen auf dem Markt zur Verfügung steht, finden individuell angefertigte Masken in der Akutmedizin so gut wie keine Anwendung.

\section{Nasenmasken}

Nasenmasken umschließen lediglich die Nase, sodass der Patient den Mund während der Beatmung aktiv geschlossen halten muss ( $\bullet$ Abb. 1 a). Da dies insbesondere während einer akuten respiratorischen Insuffizienz mit ausgeprägter Dys- und Tachypnoe oft nicht möglich ist, werden Nasenmasken nur selten zur Therapie der akuten respiratorischen Insuffizienz eingesetzt [4]. Sie werden vornehmlich zur Therapie der chronisch respiratorischen Insuffizienz im Rahmen einer außerklinischen Beatmung eingesetzt [4].

Die am häufigsten eingesetzten Nasenmasken umschließen die Nase vollständig, wenngleich Nasenmasken existieren, die den Nasenlöchern aufliegen und/oder leicht in sie hineinragen. Diese sogenannten „Nasenpillows“ finden in der Regel jedoch keinen Einsatz in der Akutmedizin und werden vornehmlich in der Schlafmedizin angewendet. Sie können allerdings bei vorhandenen Druckstellen auf dem Nasenrücken eine Alternative darstellen, wenngleich sie für den Einsatz höherer Inspirationsdrücke (z. B. > 20 mbar) nicht geeignet sind.
Ein wesentlicher Vorteil der Nasenmasken gegenüber den Vollgesichtsmasken, oder auch den Nasen-Mund-Masken, stellt die Möglichkeit des Abhustens und somit der Sekreteliminierung dar. Dies kann insbesondere bei Erkrankungen mit erhöhter Sputumproduktion, wie z.B. COPD oder Pneumonie, einen Vorteil bieten. Auch wenn eine starke Sekretproduktion als relative Kontraindikation einer nichtinvasiven Beatmung angesehen wird, kann im Einzelfall der Einsatz einer Nasenmaske bei diesen Patienten erwogen werden. Weitere Vorteile sind die Möglichkeit der Nahrungsaufnahme oder der Kommunikation während der Beatmungstherapie.

\section{Nasen-Mundmaske}

Die am häufigsten eingesetzte Maske bei der nichtinvasiven Beatmung zur Therapie der akuten respiratorischen Insuffizienz ist die Nasen-Mundmaske ( $\bullet$ Abb.1 b). Nasen-Mundmasken umschließen Nase und Mund und kommen immer dann zum Einsatz, wenn der Patient nicht in der Lage ist, den Mund zu schließen und ausschließlich durch die Nase zu atmen. Da dies bei fast allen Patienten mit akuter respiratorischer Insuffizienz der Fall ist, stellen Nasen-Mundmasken den bisher dominierenden nichtinvasiven Beatmungszugang auf der Intensivstation dar [3]. Der am häufigsten zu beachtende Nachteil ist die Beeinträchtigung der verbalen Kommunikation und die fehlende Möglichkeit zur Sekreteliminierung während der Beatmung.

Im Hinblick auf die Beatmungsqualität (Verbesserung des Gasaustausches und Verhinderung einer Intubation) gab es bei Patienten mit kardialem Lungenödem und akut-exazerbierter COPD keinen wesentlichen Unterschied zwischen Nasen- und Nasen-Mundmaske, wenngleich die Nasen-Mundmasken besser toleriert wurden [5]. Dies wurde durch eine weitere Studie untermauert, in der bei Patienten mit akut exazerbierter COPD kein Unterschied zwischen beiden Beatmungszugängen im Hinblick auf Verbesserung der Blutgase und Entlastung der Atemmuskulatur gefunden wurde [6]. Somit obliegt es dem Therapeuten anhand verschiedener Faktoren, wie Schwere der respiratorischen Insuffizienz oder aber Patientenwunsch, den entsprechenden Beatmungszugang zu wählen. Nava et al. hoben in einer Übersichtsarbeit hervor, dass der Wahl des nichtinvasiven Beatmungszuganges eine individuelle, der Situation angepasste Entscheidung vorausgehen sollte [3].

Eine in der Praxis bewährte Methode stellt der Wechsel von einer auf die andere Beatmungsmaske während der Therapie dar. Wird im Rahmen einer schweren akuten respiratorischen Insuffizienz mit einer Nasen-Mundmaske begonnen, so kann im Verlauf bei gebesserter klinischer Gesamtsituation der Wechsel auf eine Nasenmaske Vorteile im Hinblick auf Patientenkomfort und Entlastung möglicher Druckstellen mit sich bringen. Dies spielt insbesondere dann eine Rolle, wenn höhere Beatmungsdrücke verwendet werden, da höhere Beatmungsdrücke zwangsläufig höhere Anpressdrücke der Masken im Gesichtsbereich erfordern, und somit ein erhöhtes Risiko für die Entwicklung von Druckstellen besteht [7].

\section{Vollgesichtsmasken}

Ein immer häufiger eingesetzter Beatmungszugang der nichtinvasiven Beatmung stellt die Vollgesichtsmaske dar, welche das komplette Gesicht inklusive Augen und Stirn umschließt ( $\bullet$ Abb. 1 c). Da Vollgesichtsmasken nicht direkt auf dem Nasenrücken aufliegen, stellen sie eine wichtige Alternative bei drohenden oder bestehenden Druckstellen im Bereich des Nasenrückens dar. Eine neuere Studie beschreibt die erfolgreiche 

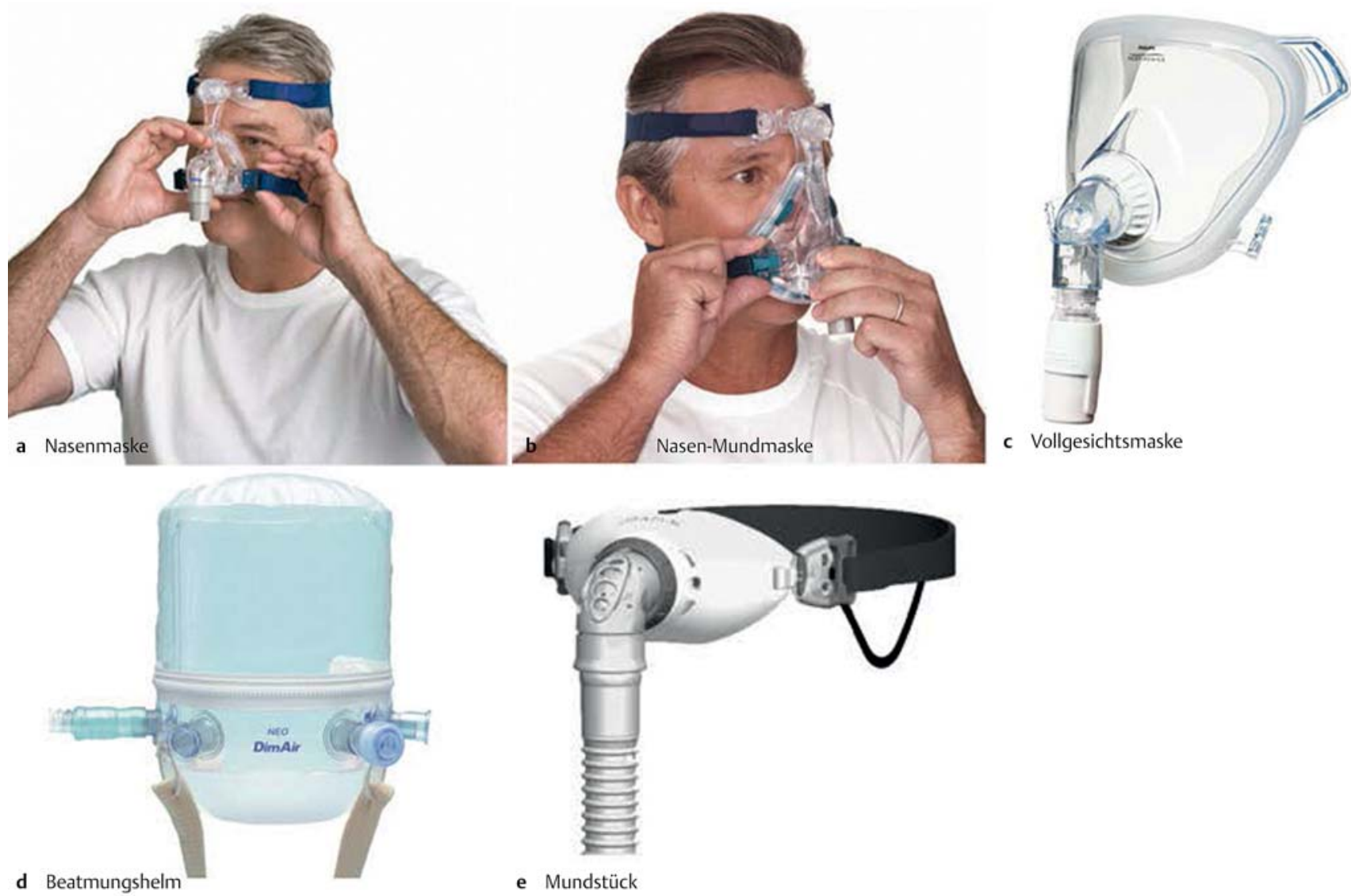

Abb. 1 Verschiedene nichtinvasive Beatmungszugänge. Mit freundlicher Genehmigung der Firmen (a und b) ResMed (c) Philips Respironics (d) Covidien (e) Fisher \& Paykel Healthcare.

Rescue-Therapie mittels Vollgesichtsmaske bei Patienten mit hyperkapnischem Lungenversagen und do-not-intubate-order, die nicht erfolgreich mit Nasen-Mundmasken behandelt werden konnten [8].

\section{Beatmungshelme}

Beatmungshelme sind transparente Zylinder, welche den gesamten Kopf umschließen und mittels Haltebändern unter den Achseln fixiert werden ( $\bullet$ Abb. 1 d). Sie bieten eine gute Alternative zu den bereits angesprochenen Interfaces, da sie nicht direkt auf der Haut im Gesichtsbereich aufliegen. Aufgrund des größeren Totraumvolumens erscheint eine effektive $\mathrm{CO}_{2}$-Elimination auf den ersten Blick erschwert, sodass der Beatmungshelm primär zur Therapie der hypoxischen respiratorischen Insuffizienz als sinnvoll erscheint. In der Tat wurde der Beatmungshelm in einigen Studien erfolgreich zur Therapie der akuten hypoxischen respiratorischen Insuffizienz eingesetzt $[9,10]$. Darüber hinaus zeigte die Studie von Antonelli und Kollegen eine bessere Therapiecompliance mit dem Beatmungshelm im Vergleich zur Kontrollgruppe, wenngleich es sich hier nicht um eine direkt vergleichende Studie, sondern um einen Vergleich mit einem historischen Kollektiv handelt [9]. Somit stellt der Beatmungshelm eine gute Alternative zur Maskentherapie im Rahmen einer akuten Hypoxie dar und eignet sich insbesondere als Alternative, wenn im Rahmen einer langen Therapiedauer die Beatmungsmaske vom Patienten nicht mehr toleriert wird. Zusätzlich können Druckstellen im Bereich des Nasenrückens verhindert oder entlastet werden, wenngleich Druckstellen im Bereich der Achseln oder des Halses nicht selten sind und beachtetet werden müssen. Hier könnte zukünftig eine neue Helmgeneration ohne Notwendigkeit der Axillenhalterung Abhilfe schaffen [11]. Der Beatmungsdruck kann von den Patienten ebenfalls als unangenehm empfunden werden. Unter der Beatmung ist insbesondere ein Druckausgleich in den Ohren erschwert und daher regelmäßig während der Therapie notwendig. Dies und auch die Geräuschbelastung unter dem Helm müssen vor Einleitung einer solchen Therapie mit den Patienten besprochen werden.

Je größer die Beatmungszugänge sind, desto größer ist auch das Totraumvolumen und somit die Gefahr der verminderten $\mathrm{CO}_{2}$ Elimination. Trotz seines großen Totraumvolumens wurde der Beatmungshelm auch zur Therapie der akuten hyperkapnischen respiratorischen Insuffizienz eingesetzt und untersucht. Es konnte gezeigt werden, dass im Rahmen einer akut hyperkapnischexazerbierten COPD eine signifikante Senkung erhöhter $\mathrm{PaCO}_{2}-$ Werte mit dem Helm erzielt werden konnte [12]. Der Vergleich mit einem historischen Kollektiv, welches mit einer NasenMundmaske behandelt wurde, zeigte jedoch, dass trotz höherer Beatmungsdrücke unter der Helmtherapie die $\mathrm{CO}_{2}$-Senkung weniger effektiv war [12]. Hinzu kommt die Tatsache, dass mit steigendem Beatmungsdruck und steigender Beatmungsfrequenz die Synchronisation des Patienten mit dem Beatmungsgerät aufgrund erschwerter Triggereigenschaften leidet [13]. Somit sollte die Indikation einer Therapie mittels Beatmungshelm im Rahmen einer hyperkapnischen respiratorischen Insuffizienz kritisch geprüft werden, wenngleich ihr Einsatz sich nicht generell verbietet. Setzt man den Beatmungshelm zur Therapie der Hyperkapnie ein, sollte auf ausreichend hohe Beatmungsdrücke geachtet werden und eine engmaschige Kontrolle der Blutgase erfolgen. 
Tab. 1 Vor- und Nachteile der häufig eingesetzten nichtinvasiven Beatmungszugänge (adaptiert nach Referenz [35]).

\begin{tabular}{|c|c|c|c|}
\hline & Nasenmaske & $\begin{array}{l}\text { Nasen-Mundmaske bzw. } \\
\text { Vollgesichtsmaske }\end{array}$ & Beatmungshelm \\
\hline Mundatmung & $\begin{array}{l}\text { nicht möglich } \\
\text { bzw. } \\
\text { ineffektive Beatmung }\end{array}$ & möglich & möglich \\
\hline Mundleckage & häufig & nicht vorhanden & nicht vorhanden \\
\hline Kommunikation & möglich & erschwert & möglich \\
\hline Exspektoration & gut möglich & erschwert & erschwert \\
\hline Totraum & gering & größer & sehr groß \\
\hline Bronchoskopie & möglich & möglich & möglich \\
\hline Druckstellen & häufig am Nasenrücken & $\begin{array}{l}\text { Nasen-Mundmaske: } \\
\text { häufig am Nasenrücken } \\
\text { Vollgesichtsmaske: } \\
\text { gute Alternative bei Druckstellen am } \\
\text { Nasenrücken }\end{array}$ & $\begin{array}{l}\text { häufig im Bereich der Haltebänder (Axilla) } \\
\text { und am Hals, aber gute Alternative bei } \\
\text { Druckstellen am Nasenrücken }\end{array}$ \\
\hline Ohrendruck & selten & selten & häufig \\
\hline Verfügbarkeit & schnell & schnell & Beatmungszugang der zweiten Wahl \\
\hline Indikation & respiratorische Insuffizienz Typ I und II & respiratorische Insuffizienz Typ I und II & primär respiratorische Insuffizienz Typ I \\
\hline
\end{tabular}

Respiratorische Insuffizienz Typ I= hypoxisch, respiratorische Insuffizienz Typ II = hyperkapnisch.

\section{Mundstücke}

Mundstücke werden aktiv von den Patienten zwischen Lippen und Zähnen festgehalten und können je nach Fabrikat auch mit Haltebändern fixiert werden ( $\bullet$ Abb.1 e). Die aktive Komponente des Patienten, also das Fixieren des Mundstückes im Mundbereich, ist jedoch unumgänglich. Mundstücke werden vor allem zur Langzeittherapie der chronisch respiratorischen Insuffizienz bei Patienten mit neuromuskulären Erkrankungen eingesetzt. Ihr Einsatz in der Akutmedizin ist relativ selten, wenngleich er sich nicht verbietet. Bei Mukoviszidosepatienten mit einer akut auf chronisch respiratorischen Insuffizienz wurden Mundstücke als alternativer nichtinvasiver Beatmungszugang eingesetzt [14].

Um bei der Verwendung von Mundstücken einem Entweichen von Luft durch die Nase vorzubeugen, können Nasenklammern verwendet werden. Dennoch sei hier erwähnt, dass Mundstücke nicht als primärer Beatmungszugang in der Akutmedizin eingesetzt werden und dies auch sicherlich nicht sinnvoll ist.

\section{Welche Maske in welcher Situation?}

Generell bietet die Nasen-Mundmaske als nichtinvasiver Beatmungszugang in der Akutmedizin wesentliche Vorteile wie z.B. das Ermöglichen einer Mundatmung und somit weniger Leckage durch den Mund, stabilere mittlere Atemwegsdrücke vor allem im Schlaf und weniger notwendige Kooperation von Seiten des Patienten. Deshalb kann prinzipiell die Nasen-Mundmaske als primärer Beatmungszugang im Rahmen einer nichtinvasiven Akutbeatmung empfohlen werden. Dennoch sollte der Wahl eines Beatmungszuganges immer eine sorgfältige Evaluierung verschiedener Faktoren, wie z.B. Wünsche und Bedürfnisse des Patienten, anatomische Besonderheiten, Beatmungsmodus und nicht zuletzt die Art der zugrunde liegenden respiratorischen Insuffizienz, vorausgehen. Diese kritische Evaluierung, insbesondere im Hinblick auf Patientenkomfort, Beatmungsqualität, Leckagen oder Druckstellen, sollte im Verlauf einer nichtinvasiven Beatmung wiederholt erfolgen, um einen notwendigen Wechsel des Beatmungszuganges frühzeitig zu erkennen und auch zu vollziehen. Vor- und Nachteile der am häufigsten verwendeten nichtinvasiven Beatmungszugänge zur Therapie der akuten respiratorischen Insuffizienz sind in Tab. 1 dargestellt.

\section{Beatmungszugänge bei invasiver Beatmung}

\section{Endotrachealtuben}

Die endotracheale Intubation gilt nach wie vor als Goldstandard zur Sicherung der Atemwege. Indikationen sind im Wesentlichen Störungen respiratorischer, kardiozirkulatorischer und/oder neurologischer Organfunktionen sowie die Durchführung einer sicheren Beatmung während einer Narkose. Zudem besteht eine absolute Intubationsindikation bei fehlenden Schutzreflexen zum Aspirationsschutz [15]. Obwohl in der Akutsituation lebensrettend, kann die Intubation und invasive Beatmung mit potenziellen Nebenwirkungen bzw. Komplikationen assoziiert sein. Die zugrundeliegenden pathophysiologischen Mechanismen hierfür sind der beatmungsinduzierte Lungenschaden (ventilator induced lung injury, VILI), der beatmungsindizierte Zwerchfellschaden (ventilator-induced diaphragmatic dysfunction, VIDD), die ventilatorassoziierte Pneumonie (VAP) sowie die Nebenwirkungen der notwendigen Analgesie und Sedierung. Daher wird heute bei notwendiger Beatmung, wenn die Indikationen für eine nichtinvasive Beatmung gegeben sind und keine Kontraindikationen vorliegen, die nichtinvasive Beatmung bevorzugt [16]. Die Intubation kann auf oralem (orotracheale Intubation) oder nasalem Weg (nasotracheale Intubation) erfolgen, wobei die nasotracheale Intubation aufgrund der höheren Inzidenz tubusinduzierter Sinusitiden [17] mittlerweile im Intensivbereich kaum noch angewendet wird. Mechanische Komplikationen durch den Tubus selbst sind durch verbesserte Tubuseigenschaften in den Hintergrund getreten. "High volume/low-pressure"Cuffs sind heute Standard und führen seltener zu Schädigungen der Trachealwand ( $\mathbf{A b b}$.2). Eine ausreichende Cuff-Füllung der Niederdruckmanschette ist üblicherweise bei $20-25 \mathrm{~cm} \mathrm{H}_{2} \mathrm{O}$ erreicht, höhere Drücke können zu Mukosaschädigungen führen und sollten vermieden werden, der verwendete Cuffdruck muss regelmäßig, mindestens $1 \times$ pro Schicht, kontrolliert werden.

Um den Atemwegswiderstand insbesondere bei Spontanatmung so weit wie möglich zu reduzieren, sollte der größtmögliche Tubus verwendet werden, der ohne Widerstand in die Trachea vorgeschoben werden kann. Der Einsatz von Endotrachealtuben (und Trachealkanülen) mit der Möglichkeit zur subglottischen Absaugung kann besonders bei längeren Beatmungszeiten erwo- 


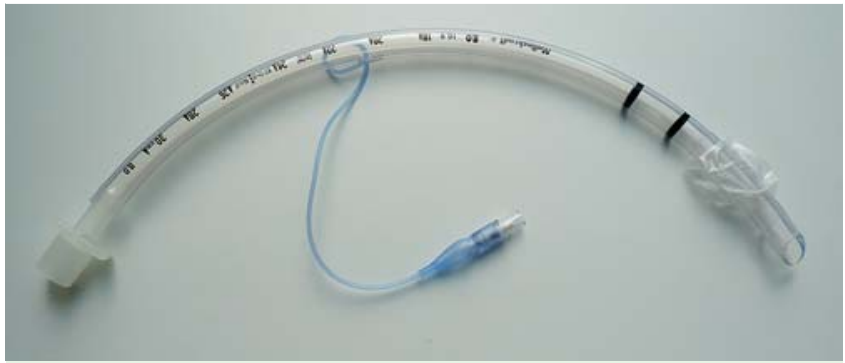

Abb. 2 Standard-Endotrachealtubus mit high volume/low-pressure-Cuff (Fa. Mallinckrodt).

gen werden, da diese laut einigen Studiendaten mit geringeren Pneumonieraten assoziiert sind [18] ( Abb.3a,b). Allerdings sind diese Devices im Moment noch relativ kostenintensiv.

Eine Innovation der letzten Jahre im Bereich des Airway-Managements sind Videolaryngoskope oder optische Laryngoskope. Laut aktueller Datenlage kann dadurch eine bessere Visualisierung der Glottisregion ermöglicht werden. Diese Geräte führten in den meisten Studien zu einem höheren Intubationserfolg [19]. Trotz der im Vergleich zur konventionellen Laryngoskopie steileren Lernkurve sind ein umfangreiches Training und klinische Erfahrung jedoch unabdingbar.

Die gravierendste Komplikation einer nicht möglichen oder fehlerhaften Intubation ist die Hypoxie mit Todesfolge oder persistierenden neurologischen Defiziten. Hauptfaktoren für Komplikationen sind suboptimale Technik, fehlende Erfahrung sowie das Vorhandensein eines schwierigen Atemweges. Per se ist die Intubation auf der Intensivstation beim kritisch kranken Patienten, verglichen mit der elektiven Einleitung im OP, eine „HighRisk-Prozedur“. Daher kommt der notwendigen Ausbildung des Personals, der adäquaten Ausstattung und dem Monitoring eine große Bedeutung zu. Explizit empfohlen wird die Verwendung der Kapnografie bei jeder Intubation [20]. Zudem kann das Vorhandensein einer Intubations-Checkliste die Inzidenz von Atemwegskomplikationen bei der Intubation auf der Intensivstation reduzieren [21].

\section{Koniotomie}

Bei dieser Form der Atemwegssicherung wird das Ligamentum cricothyroideum, das sich zwischen Schild- und Ringknorpel befindet, notfallmäßig durchtrennt und eine Kanüle oder ein Tubus in die Trachea eingeführt. Die Koniotomie ist eine Notfallmaßnahme beim schwierigen Atemweg und fehlender konventioneller Intubationsmöglichkeit. Die Intervention kann sowohl konventionell chirurgisch, aber auch als Punktionsverfahren in Seldinger-Technik durchgeführt werden. Entsprechende Sets sollten auf der Intensivstation im Notfallwagen vorgehalten werden.

\section{Tracheotomie}

Die Tracheotomie ist der Standardzugang, wenn eine Langzeitbeatmung beim Intensivpatienten absehbar wird. Die Indikation ergibt sich vorwiegend aus den Nachteilen der endotrachealen Intubation. In der Vergangenheit war ein Hauptargument für die Tracheotomie die Vermeidung von Larynxschäden durch den Orotrachealtubus. Aufgrund verbesserter Tubuseigenschaften ist diese Problematik jedoch etwas in den Hintergrund getreten. Da sich in Studien kein konsistenter Effekt für eine Frühtracheotomie nachweisen ließ, sollte eine Tracheotomie im Regelfall frühestens nach zwei Wochen Beatmungsdauer erwogen werden

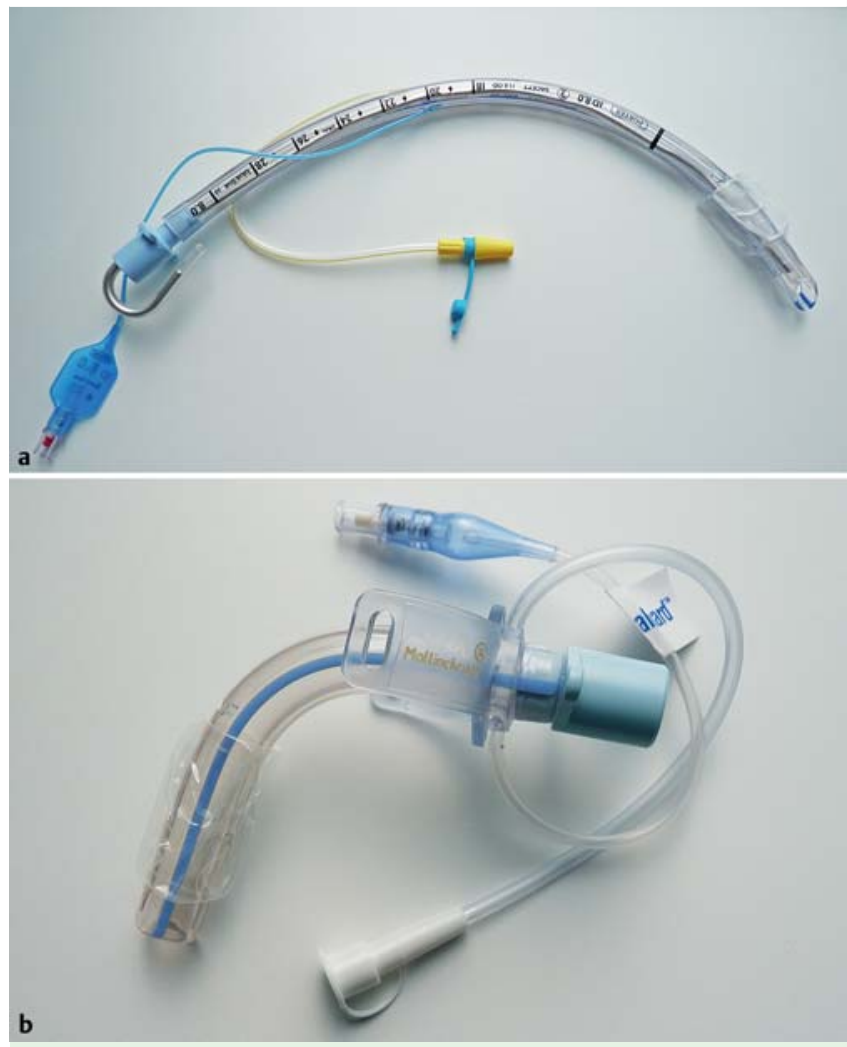

Abb. 3 a Endotrachealtubus mit der Möglichkeit zur subglottischen Absaugung (Fa. Portex). b Trachealkanüle mit der Möglichkeit zur subglottischen Absaugung (Fa. Mallinckrodt).

[22]. Hierbei müssen immer die Nachteile einer längeren Intubationszeit auf der einen und die prozeduralen Risiken der Tracheotomie auf der anderen Seite abgewogen werden. Beachtet werden muss, dass die Abschätzung der Beatmungsdauer selbst für Experten im Einzelfall schwierig sein kann. Im Vergleich zum translaryngealen Endotrachealtubus wird die Trachealkanüle besser toleriert und erlaubt im Regelfall die Reduktion von Sedativa und Analgetika. Der Wegfall eines Hindernisses im Mundraum ermöglicht zudem eine bessere Pflege des Nasen-RachenBereiches und eine frühere enterale Ernährung. Weiterhin wird dadurch die Bronchialtoilette erleichtert. Zur Kommunikationserleichterung sind spezielle Sprechventile, die auf die Trachealkanüle gesetzt werden, sowie einsetzbare Sprechkanülen verfügbar. All diese Faktoren tragen zu einem erhöhten Patientenkomfort bei. Angeführte atemphysiologische Argumente für die Erleichterung des Weanings durch eine Tracheotomie im Vergleich zur fortgesetzten endotrachealen Intubation sind u.a. ein reduziertes Totraumvolumen, eine Verringerung des Atemwegswiderstandes sowie der Atemarbeit. Allerdings sind die Effekte nur gering und abhängig vom Lumen der gewählten Trachealkanüle. In den letzten Jahren hat sich die perkutane Dilatationstracheotomie aufgrund einiger Vorteile zum Verfahren der ersten Wahl beim Intensivpatienten entwickelt [23]. Allerdings müssen die spezifischen Kontraindikationen der perkutanen Technik beachtet werden ( Tab.2) [24,25].

Eine operative Tracheotomie sollte auch angestrebt werden, wenn ein stabiles permanentes Tracheostoma bei außerklinischer Beatmung erforderlich ist. Dilatationstracheostomata sind wegen Schrumpfungstendenz und Risiko einer Kanülenfehllage nur in Ausnahmefällen zu akzeptieren [26]. 
Tab. 2 Kontraindikationen der perkutanen Dilatationstracheotomie erschwerte oder unmögliche Intubationsverhältnisse unklare Anatomie mit nicht identifizierbarem Trachealverlauf instabile Halswirbelsäulenfraktur nicht korrigierbare Gerinnungsstörungen Atemwegsnotfall

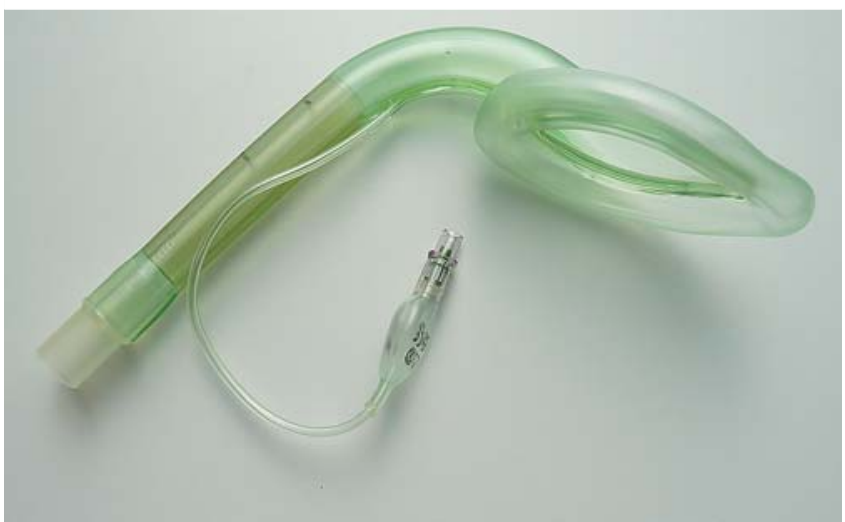

Abb. 4 Larynxmaske (Fa. Ambu).

Derzeit stehen sechs Methoden der perkutanen Dilatationstracheotomie zur Verfügung. Für alle Verfahren gilt als Prinzip die perkutane Nadelpunktion der Trachea und Platzierung eines Führungsdrahts und nachfolgende Anlage einer Tracheotomie mithilfe von Dilatationsinstrumenten [27]. Die Rate der klinisch relevanten Komplikationen ist für alle Verfahren gleichermaßen niedrig, allerdings wird die Ein-Schritt-Dilatationstechnik bzw. modifizierte Ciaglia-Technik aufgrund der einfachen, schnellen Technik mittlerweile in Deutschland und weltweit am häufigsten eingesetzt [28]. Wesentliche Sicherheitsmaßnahmen bei der Durchführung der perkutanen Dilatationstracheotomie zur Vermeidung von Komplikationen sind unter anderem die Kenntnis der Halsanatomie, die Beachtung aller Kontraindikationen sowie die konsequente videobronchoskopische Visualisierung des Eingriffs durch einen erfahrenen Untersucher [29].

\section{Supraglottische Atemwegshilfen}

In der Notfallmedizin sowie in der Anästhesie werden supraglottische Atemwegshilfen wie Larynxmaske ( Abb.4), Larynxtubus und Combitubus mittlerweile zunehmend häufiger eingesetzt [30]. Auf der Intensivstation ist der Einsatz beim Management des schwierigen Atemweges und nicht erfolgreich durchführbarer endotrachealer Intubation sinnvoll. Allerdings bietet die Larynxmaske keinen sicheren Aspirationsschutz, Voraussetzung für die zielgerichtete Anwendung ist in jedem Fall eine adäquate Schulung [31].

Zudem wurde der Einsatz von Larynxmasken als Alternative zum Endotrachealtubus bei der Dilatationstracheotomie in verschiedenen Studien untersucht. Überwiegend zeigt sich eine bessere Visualisierung der intratrachealen Strukturen. Allerdings wird durch die Extubation ein sicherer Atemweg entfernt, sowohl Atemwegsverluste als auch schwierige Beatmungsbedingungen über die Larynxmaske mit Leckagen und konsekutiver Reintubation wurden beschrieben. Aufgrund der methodischen Probleme kann zurzeit keine generelle Empfehlung für den Einsatz von Larynxmasken als bevorzugte Atemwegssicherung während einer Dilatationstracheotomie ausgesprochen werden [32].

\section{Physiologie der Beatmungszugänge}

Sowohl für die invasiven als auch für die nichtinvasiven Beatmungszugänge sind nicht-lineare flussabhängige Widerstände (Rohrer-Gleichung) beschrieben, die stark vom jeweiligen Beatmungszugang abhängig sind [33]. Bei Endotrachealtuben und Trachalkanülen ist insbesondere bei laminarem Flow (Spontanatmung) der Durchmesser der künstlichen Atemwege für die Atemarbeit relevant. Zudem weisen Endotrachealtuben gegenüber Trachealkanülen gleichen Durchmessers einen höheren Widerstand auf, da sie länger sind. Aus den genannten Gründen sollte zur Reduktion des Atemwegswiderstandes jeweils der größtmögliche Tubus bzw. die größtmögliche Trachealkanüle ausgewählt werden. Bei der nichtinvasiven Beatmung scheinen die flussabhängigen Widerstände eine eher untergeordnete Rolle zu spielen. Es konnte in einer Laborstudie gezeigt werden, dass zwar ein nicht-linearer, flussabhängiger Druckabfall in der Nasenmaske besteht, dieser aber gering und nicht abhängig von der Größe der verwendeten Maske ist. Die flussabhängigen Widerstände beim nichtinvasiven Beatmungszugang scheinen im Wesentlichen von der Gänsegurgel abhängig zu sein [34].

\section{Interessenkonflikt}

M. Dreher gibt an, dass kein Interessenkonflikt besteht.

S. Kluge hat eine finanzielle Unterstützung von der Fa. Cook für eine Studie erhalten.

\section{Literatur}

1 Zilberberg MD, de Wit M, Shorr AF. Accuracy of previous estimates for adult prolonged acute mechanical ventilation volume in 2020: update using 2000 - 2008 data. Crit Care Med 2012; 40: 18 - 20

2 Evans TW. International Consensus Conferences in Intensive Care Medicine: non-invasive positive pressure ventilation in acute respiratory failure. Organised jointly by the American Thoracic Society, the European Respiratory Society, the European Society of Intensive Care Medicine, and the Société de Réanimation de Langue Française, and approved by the ATS Board of Directors, December 2000. Intensive Care Med 2001; 27: 166-178

3 Nava S, Navalesi P, Gregoretti C. Interfaces and humidification for noninvasive mechanical ventilation. Respir Care 2009; 54: 71 - 84

4 Elliott MW. The interface: crucial for successful noninvasive ventilation. Eur Respir J 2004; 23: 7-8

5 Kwok H, McCormack J, Cece R et al. Controlled trial of oronasal versus nasal mask ventilation in the treatment of acute respiratory failure. Crit Care Med 2003; 31: 468-473

6 Antón A, Tárrega J, Giner J et al. Acute physiologic effects of nasal and full-face masks during noninvasive positive-pressure ventilation in patients with acute exacerbations of chronic obstructive pulmonary disease. Respir Care 2003; 48: 922 - 925

7 Dellweg $D$, Hochrainer $D$, Klauke $M$ et al. Determinants of skin contact pressure formation during non-invasive ventilation. J Biomech 2010; 43: $652-657$

8 Lemyze M, Mallat J, Nigeon $O$ et al. Rescue therapy by switching to total face mask after failure of face mask-delivered noninvasive ventilation in do-not-intubate patients in acute respiratory failure. Crit Care Med 2013; 41: $481-488$

9 Antonelli M, Conti G, Pelosi P et al. New treatment of acute hypoxemic respiratory failure: noninvasive pressure support ventilation delivered by helmet-a pilot controlled trial. Crit Care Med 2002; 30: 602 - 608

10 Cosentini R, Brambilla AM, Aliberti $S$ et al. Helmet continuous positive airway pressure vs oxygen therapy to improve oxygenation in community-acquired pneumonia: a randomized, controlled trial. Chest 2010; 138: $114-120$

11 Olivieri C, Costa R, Spinazzola G et al. Bench comparative evaluation of a new generation and standard helmet for delivering non-invasive ventilation. Intensive Care Med 2013; 39: 734-738 
12 Antonelli $M$, Pennisi MA, Pelosi $P$ et al. Noninvasive positive pressure ventilation using a helmet in patients with acute exacerbation of chronic obstructive pulmonary disease: a feasibility study. Anesthesiology 2004; 100: 16-24

13 Moerer O, Beck J, Brander L et al. Subject-ventilator synchrony during neural versus pneumatically triggered non-invasive helmet ventilation. Intensive Care Med 2008; 34: 1615-1623

14 Madden BP, Kariyawasam H, Siddiqi AJ et al. Noninvasive ventilation in cystic fibrosis patients with acute or chronic respiratory failure. Eur Respir J 2002; 19: 310-313

15 Pothmann W, Kluge S. Endotracheal intubation. Dtsch Med Wochenschr 2010; 135: $94-97$

16 Schönhofer B, Kuhlen R, Neumann P et al. Non-invasive ventilation as treatment for acute respiratory insufficiency. Essentials from the new S3 guidelines. Anaesthesist 2008; 57: 1091 - 1102

17 Deutschman CS, Wilton P, Sinow J et al. Paranasal sinusitis associated with nasotracheal intubation: a frequently unrecognized and treatable source of sepsis. Crit Care Med 1986; 14: 111-114

18 Lacherade J-C, De Jonghe B, Guezennec $P$ et al. Intermittent subglottic secretion drainage and ventilator-associated pneumonia: a multicenter trial. Am J Respir Crit Care Med 2010; 182: 910-917

19 Pirlich N, Piepho T, Gervais H et al. Indirect laryngoscopy/video laryngoscopy. A review of devices used in emergency and intensive care medicine in Germany. Med Klin Intensiv Notfallmedizin 2012; 107: $521-530$

20 Cook T, Behringer EC, Benger J. Airway management outside the operating room: hazardous and incompletely studied. Curr Opin Anaesthesiol 2012; 25: 461-469

21 Jaber S, Jung B, Corne P et al. An intervention to decrease complications related to endotracheal intubation in the intensive care unit: a prospective, multiple-center study. Intensive Care Med 2010; 36: 248 255

22 Wang $F, W u Y, B o L$ et al. The timing of tracheotomy in critically ill patients undergoing mechanical ventilation: a systematic review and meta-analysis of randomized controlled trials. Chest 2011; 140: $1456-1465$

23 Higgins KM, Punthakee X. Meta-analysis comparison of open versus percutaneous tracheostomy. Laryngoscope 2007; 117: 447-454

24 Braune S, Kluge S. Update on tracheotomy. Med Klin Intensiv Notfallmedizin 2012; 107: $543-547$

25 Freeman $B D$, Morris $P E$. Tracheostomy practice in adults with acute respiratory failure. Crit Care Med 2012; 40: 2890-2896

26 Windisch W, Walterspacher S, Siemon K et al. Guidelines for non-invasive and invasive mechanical ventilation for treatment of chronic respiratory failure. Published by the German Society for Pneumology (DGP). Pneumol Stuttg Ger 2010; 64: 640-652

27 Braune S, Kluge S. Percutaneous dilatational tracheostomy. Dtsch Med Wochenschr 2011; 136: 1265 - 1269

28 Kluge S, Baumann HJ, Maier C et al. Tracheostomy in the intensive care unit: a nationwide survey. Anesth Analg 2008; 107: 1639-1643

29 Baumann HJ, Kemei C, Kluge S. Tracheostomy in the intensive care unit. Pneumol 2010; 64: 769-776

30 Timmermann A, Byhahn C, Wenzel $V$ et al. Handlungsempfehlung für das präklinische Atemwegsmanagement. Anästh Intensiv 2012; 53 : $294-308$

31 Schwarzkopf K. Supraglottic airway devices. Med Klin Intensiv Notfallmedizin 2012; 107: 531-536

32 Pratt T, Bromilow J. Laryngeal mask airway for airway control during percutaneous dilatational tracheostomy. Anaesth Intensive Care 2011; 39: $1009-1013$

33 Guttmann J, Eberhard L, Fabry B et al. Continuous calculation of intratracheal pressure in tracheally intubated patients. Anesthesiology 1993; 79: $503-513$

34 Windisch W, Buchholt A, Stahl CA et al. Flow-dependent resistance of nasal masks used for non-invasive positive pressure ventilation. Respirol Carlton Vic 2006; 11: 471 - 476

35 Schönhofer B. Nichtinvasive Beatmung - gestern, heute und morgen. Intensivmed 2008; 45: 182-193 